\title{
ULTRASONOGRAPHIC SCORING INDEX CAN BE USEFUL IN \\ THE PREDICTION OF THYROID MALIGNANCY IN \\ SUBCENTIMETER AND SUPRACENTIMETER THYROID \\ NODULES
}

\section{Neslihan CUHACl ${ }^{1}$, Berna OGMEN ${ }^{2}$, Ali TAM${ }^{1}$, Cevdet AYDIN ${ }^{1}$, Oya TOPALOGLU ${ }^{1}$, Aylin KILIC YAZGAN 3 ,}

Gurkan DUMLU', Reyhan ERSOY', Bekir CAKIR ${ }^{1}$

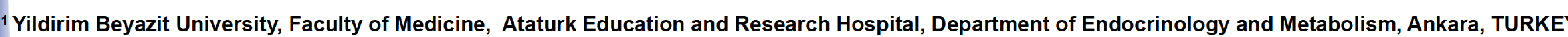
2 Ataturk Education and Research Hospital, Department of Endocrinology and Metabolism, Ankara, TURKEY ${ }^{3}$ Ataturk Education and Research Hospital, Department of Pathology, Ankara, TURKEY

${ }^{4}$ Yildirim Beyazit University, Faculty of Medicine, Ataturk Education and Research Hospital, Department of GeneralSurgery, Ankara, TURKEY

\section{INTRODUCTION}

$>$ The increased rate of thyroid malignancy as well as incidental and subcentimeter thyroid nodules have been attributed to increasing use of high-resolution US which can detect the non-palpabl or subcentimeter (maximum diameter $\leq 1 \mathrm{~cm}$ ) thyroid nodules.

$>$ We aimed to evaluate the sonographic features of the tyroid nodules between $\leq 1 \mathrm{~cm}$ and $>1 \mathrm{~cm}$ according the histopathology results and to determine the ultrasonographical predictive factors for malignancy and an ultrasonographic score according the sonographic features to avoid unnecessary fineneedle aspiration biopsy (FNAB).

\section{METHODS}

$>$ We retrospectively evaluated 2233 nodules of 1118 patients who underwent thyroidectomy.

$>$ Predictive factors for distinguishing between malignant and benign histopathologic results according the ultrasonographic features were evaluated by multivariate logistic regression analysis.

> Multiple binary logistic regression with the forward logistic regression method was used to develop the formula for recommending sonographically guided biopsy.
$>$ Among the 2233 nodules 337 nodules were in the $L 1$ $\mathrm{cm}$ (group 1), 1896 were in the $>1 \mathrm{~cm}$ (group 2).

$>$ According the histopathological results, in group 1; 173 nodules were in the benign, 164 nodule were in the malignant group. Whereas in group 2; 1423 nodules were in the benign, 473 nodules were in the malignant group.

$>$ In group $1, \mathrm{AP} / \mathrm{T} \geq 1$, the presence of microcalcification, macrocalcification and hypoechoic pattern were significantly higher in the malignant group and in group 2, the presence of microcalcification, macrocalcification, hypoechoic and iso-hypoechoic pattern, solid texture, peripheral and intranodular vascularization pattern were significantly higher in the malignant group.

$>$ In group 1, the best ultrasonographic index score was found $>2$, whereas in group 2 the it was found $>4$.

Table. Index scores related with US features that can predict malignancy in nodules $\leq 1 \mathrm{~cm}$ and $>1 \mathrm{~cm}$

\begin{tabular}{lcc}
\hline & \multicolumn{2}{c}{ Nodule size } \\
& $\leq \mathbf{~ c m}$ & $>\mathbf{~} \mathbf{~ m}$ \\
Ultrasonographic ind ex score & & \\
Benign & $2.09 \pm 1.19$ & $3.97 \pm 1.46$ \\
Malignant & $3.04 \pm 1.06$ & $4.95 \pm 1.70$ \\
ROC an alysis & & \\
Area under the curve & 0.722 & 0.665 \\
$9 \% 5$ Confidence interval & $0.667-0.777$ & $0.636-0.693$ \\
$p$-value & $<\mathbf{0 . 0 0 1}$ & $<\mathbf{0 . 0 0 1}$ \\
The best cut-off point & $>2$ & $>4$ \\
Sensitivity & $68.6 \%$ & $58.1 \%$ \\
Specifity & $66.5 \%$ & $66.6 \%$ \\
$P P V$ & $64.4 \%$ & $36.7 \%$ \\
$N P V$ & $70.6 \%$ & $82.7 \%$ \\
\hline
\end{tabular}

\section{CONCLUSION}

$>$ Our US scoring may lead to clinicians and surgeons to diagnose thyroid malignancy more accurately and to select the nodules for FNAB especially in subcentimeter nodules. 